\title{
$1 \quad$ Labeling subcellular structures in living specimens using live-cell incompatible dyes with excellent optical properties
}

3 Yubing Han, ${ }^{1}$ Zhimin Zhang, ${ }^{1}$ Wenjie Liu, ${ }^{1}$ Yuanfa Yao, ${ }^{2}$ Yuchen Chen, ${ }^{1}$ Xin Luo, ${ }^{1}$ Wensheng Wang, ${ }^{1}$ Yingke $\mathrm{Xu},{ }^{2} \mathrm{Xu} \mathrm{Liu},{ }^{1,3,4, *}$ Cuifang Kuang, ${ }^{1,3,4, *} \mathrm{Xiang} \mathrm{Hao}^{1, *}$

1 State Key Laboratory of Modern Optical Instrumentation, College of Optical Science and Engineering, Zhejiang University, Hangzhou, Zhejiang 310027, China

2 Department of Biomedical Engineering, Key Laboratory of Biomedical Engineering of Ministry of Education, Zhejiang Provincial Key Laboratory of Cardio-Cerebral Vascular Detection Technology and

9 Medicinal Effectiveness Appraisal, Zhejiang University, Hangzhou, Zhejiang 310027, China

3 Ningbo Research Institute, Zhejiang University, Ningbo 315100, China

4 Collaborative Innovation Center of Extreme Optics, Shanxi University, Taiyuan 030006, China

*Correspondence and requests for materials should be addressed to X. Hao (email: haox@zju.edu.cn), C. Kuang (email: cfkuang@zju.edu.cn), and X. Liu (email: liuxu@zju.edu.cn)

Despite the urgent needs of imaging living specimens for cutting-edge biological research, most of the existing fluorescent labeling methods suffer from either poor optical properties or complicated operations to realize cell-permeability and specificity. Here, we introduce a method to overcome this tradeoff by incubating living cells and tissues with fluorescent dyes, no matter if they are cell-permeable or not, at particular conditions (concentration and temperature) without physical cell-penetration or chemical modifications. Based on this method, the mitochondrial labeling capability of Atto dyes, especially Atto $647 \mathrm{~N}$ with extraordinary optical properties, together with interesting interactions between organelles is revealed. These results indicate the great potential of using dyes, which are normally considered "live-cell incompatible", to capture the morphology and dynamics of subcellular structures in living specimens.

\section{Introduction}


Biologists rely on an array of fluorescent microscopy to observe morphologies and dynamics in living cells, which is crucial in interpreting vital physiological and pathological activities. However, although dramatic improvements have been implemented since the seminal discovery of fluorophores and their applications in microscopy $(1,2)$, it is still quite challenging to achieve live-cell specific staining simultaneously with high brightness and photostability.

In fact, most live-cell labeling methods, including fluorescent proteins (3), chemical tag techniques using cell-permeable fluorescent dyes (e.g., SNAP-Cell 647-SiR (4)), and live-cell organic fluorescent probes (e.g., MitoTracker dyes (5)), suffer from relatively low brightness and photostability (6,7). Poor optical properties of the fluorescent probes may lead to low signal-to-noise ratios (SNR) and fast photobleaching. Lower SNR reduces the quality of the microscope images or even introduce artifacts. Worse still, the photobleaching is undesirable in the long-term experiments. A dosage increase can partially remedy this problem, but it may also lead to non-specific labeling $(7,8)$. Another option is to increase the phototoxicity. This issue stands out especially in the case of imaging methods for more information in multiple dimensions (e.g., high spatiotemporal resolution, long acquisition times, and large volume imaging) $(6,9,10)$.

In the past few decades, various types of commercially available dyes, with much higher brightness and photostability than most of the live-cell probes, have been developed and widely used in fixed cells and organisms (11). However, most of these fluorophores were supposed to be "live-cell compatible", and their intracellular cytosolic delivery and specifically labeling rely heavily on specific physical or chemical methods, such as microinjection, encapsulating vesicles, or chemical modifications using cellpenetrating peptides $(7,12,13)$.

To resolve this dilemma, we present a novel strategy to label various types of subcellular structures both in living cells and in tissues using the bright and photostable dyes, which normally require complex delivery methods for subcellular labeling. Specifically, by incubating with living specimens at 1.5-15 $\mu \mathrm{M}$ for $30 \mathrm{~min}$ at $37^{\circ} \mathrm{C}$ or $20^{\circ} \mathrm{C}$, the "live-cell incompatible" dyes can also be live-cell compatible. With multiple applications using our labeling strategy, these dyes (e.g., Atto 647N) shows great potential as a live-cell mitochondrial marker with excellent optical properties. 


\section{Results}

\section{Evaluation of different dyes for labeling living cells.}

Several types of fluorescent dyes were characterized according to their structure series.

Many cell-permeable cationic dyes (e.g., Rhodamine and Carbocyanine derivates) have been developed as mitochondrial probes, as they tend to accumulate in the mitochondrial matrix driven by the potential gradient in mitochondria at about $100-\mathrm{nM}$ concentrations $(5,14,15)$. Endoplasmic reticulum (ER) staining can also be realized by raising the dosages of these dyes (7). However, for the "live-cell compatible" dyes, concentrations at the 100-nM level are not enough.

Following our previous work (7), herein, aliquots of the indicated dyes were dissolved in Dimethyl sulfoxide (DMSO) to make 3-mM stock solutions, and these solutions were diluted with phenol red-free medium to $1.5-15 \mu \mathrm{M}$ concentrations (DMSO/incubation buffer $=1 / 2,000$ ) before use. After incubation with living U2OS (human osteosarcoma cell line) cells for $30 \mathrm{~min}$ at a proper temperature, a solution of Trypan blue (TB; $1 \mathrm{mg} \cdot \mathrm{mL}^{-1}$ in PBS) was added to quench the fluorescence from the probes outside the cells $(7,16,17)$.

Firstly, four Rhodamine derivatives, i.e., Atto 495, Atto 565, Atto 590, and Atto 647N NHydroxysuccinimide (NHS) esters, were investigated due to their relatively good optical properties in the corresponding spectral bands (Fig. 1 and figs. S1-6). When incubated at $37^{\circ} \mathrm{C}$, which is the optimum temperature for cell culture, both line-shaped and bright dot signals inside the cells were observed (fig. $\mathrm{S} 1 \mathrm{~B}$ ); whereas when the incubation temperature was set to $20^{\circ} \mathrm{C}$ (room temperature), the dot signals were dramatically reduced (fig. S1B). Mitochondrial staining was confirmed by colocalization experiments (Pearson correlation coefficient: 0.70, 0.57, and 0.82 for Atto 495, Atto 590, and Atto $647 \mathrm{~N}$, respectively; Fig. 1A-C and fig. S2), indicating their direct penetration through the plasma membrane. Besides, the dot signals were partially colocalized with endocytic-unassociated vesicles (early endosomes, late endosomes, and lysosomes; Figs. 1D-I and fig. S3-5), while no colocalization was found with other vesicular structures (fig. S6). Since the extracellular fluorescence (e.g. dye aggregates) was quenched by $\mathrm{TB}$, these results indicate that (i) the dyes revealed by dot signals mainly entered living cells by endocytosis; (ii) incubation at relatively low temperature (e.g., $20^{\circ} \mathrm{C}$ ) can 
effectively reduce endocytosis of these dyes so that they can mainly enter living cells through free diffusion based on the concentration gradient.

Then, as Carbocyanine dyes, anionic Alexa Fluor (AF) 647, zwitterionic Cy3B and cationic Cy5 were chosen as representative research objects (fig. S7A-C). No mitochondrial staining was found after the incubation at either $37^{\circ} \mathrm{C}$ or $20^{\circ} \mathrm{C}$. For $\mathrm{AF} 647$ and $\mathrm{Cy} 3 \mathrm{~B}$, the introduction of sulfonic acid groups improves fluorescence and solubility in water but adds negative charges, preventing their affinity for mitochondria (18). For Cy5, the especially high hydrophobicity leads to its strong signals in the plasma membrane (fig. S7B), preventing its permeation into living cells (19).

Unexpectedly, when incubated at the same concentrations as that for the above dyes, zwitterionic BODIPY 650/665 labeled not only mitochondria but also the ER (fig. S7D), suggesting its higher cellpermeability than the other dyes tested. Our results indicate the potential of the BODIPY dyes as livecell mitochondrial (or ER) markers, breaking through the early conclusion (14) that this purpose can only be achieved using cationic dyes.

In view of these observations, we hypothesize that without additional vector reagents and physical penetration, a group of fluorescent dyes, no matter if they were initially considered to be live-cell compatible or not, can mark subcellular structures in living cells using our labeling strategy (Table 1).

\section{Labeling in various types of living cells.}

We also used the living HeLa (human adenocarcinoma cell line), HEK293 (human embryonic kidney epithelial cell line), NIH/3T3 (mouse embryonic fibroblast cell line) cells and primary cultural astrocytes from 1-day-old Sprague-Dawley (SD) rat (Fig. 2 and fig. S8). Notably, while the transfection in primary cultural astrocytes is challenging to achieve, the incubation with fluorescent probes is quite simple and efficient. Mitochondrial hyperfusion, which occurs upon cellular stresses and confers stress resistance (20), in astrocytes were captured (Fig. 2B-E and Movie S1). Some mitochondria moved radially (the direction is highlighted by the white arrowheads in Fig. 2D), and then either fused with other mitochondria (the blue arrowheads in Fig. 2D) or straighten out again (the yellow arrowheads in Fig. 2E). These dynamics may explain how mitochondrial hyperfusion is formed.

\section{Cell viability after labeling.}


Although raising the incubation concentrations helps the above dyes to permeate into living cells, it may also cause cytotoxicity from both the dyes themselves and the organic solvent in the incubation buffer (e.g., DMSO). To further confirm that our approach is compatible with the living specimens, the cytotoxicity of this method was then investigated by 3-(4,5-dimethylthiazol-2-yl)-5-(3carboxymethoxyphenyl)-2-(4-sulfophenyl)-2H-tetrazolium (MTS) assay, which is a colorimetric method for determining the number of viable cells in proliferation or cytotoxicity assays (21). In living cells, the MTS tetrazolium compound can be converted into a colored formazan product that is soluble in the tissue culture medium, and the quantity of formazan product as measured by absorbance at $490 \mathrm{~nm}$ is directly proportional to the number of living cells in culture (22). After incubation with the indicated dyes at $37^{\circ} \mathrm{C}$ for $30 \mathrm{~min}$ (cells were untreated in the control groups), the cells were incubated with MTS reagents for another $3 \mathrm{~h}$ according to the instructions. The cell viabilities were expressed as the percentage of the absorbance of the dye-treated cells (after subtracting the values of the dyes in the cells) to the untreated controls, and all of the measurements were performed in triplicate. The results showed that after treated by the indicated dyes, the cell viabilities of the above five types of cells were usually higher than $80 \%$, except in isolated cases (fig. S9). The cell viability higher than 100\% (especially obvious for Atto 565 that enters the living cells mainly by endocytosis at $37^{\circ} \mathrm{C}$ ) may result from the involvement of cellular redox factors in endocytosis (23). The result indicates the low cytotoxicity of our method.

\section{Optical properties of Atto 647N.}

We quantitatively compared the optical properties of the frequently-used red-absorbing fluorophores. In terms of brightness, Atto $647 \mathrm{~N}$ exhibits much better performance than the $\mathrm{SiR}$ dye (4) and MitoTracker Deep Red (5) (Table S1). To evaluate photostability, fluorescence intensity curves were extracted from 20-min confocal imaging of U2OS cells (Fig. 3A and fig. S10), where all the imaging settings (laser power, size of field-of-view, imaging speed, etc.) were retained to control variables (Table S2). The results suggest that Atto 647N exhibited the best photostability (Fig. 3A and fig. S10A). Besides, by adding antioxidant reagent Prolong Live (24), all the bleaching speeds of these dyes decelerated to some extent. However, the difference between the dyes remained unchanged (Fig. $3 \mathrm{~A})$. The performance of Atto $647 \mathrm{~N}$ was further investigated using stimulated emission depletion (STED) microscopy (Fig. 3B-E). The distance of mitochondrial outer membranes was resolved as 116 $\mathrm{nm}$ in STED mode, while the confocal image showed blurred structures without fine structures (Fig. 3C, 
138 D). In addition, structures of folded inner membranes, cristae, which were arranged in groups, and the 139 voids between them were visible in the STED image (white arrows in Fig. 2E) (7,25,26). Therefore, 140 these results indicate that applying our method in live-cell mitochondrial staining, especially in long141 term or super-resolution imaging, Atto 647N can substitute SiR dye by offering better optical properties.

Dual-color staining combined with either fluorescent proteins (EMTB-3XGFP (27); Fig. 4A) or other probes (ER-Tracker Green; Fig. 4B-D and fig. S11) were performed, suggesting the compatibility the ER substructures colocalized with mitochondria over time (the white and yellow arrowheads in Fig. 4B and fig. S11A). The possibility of crosstalk between channels was ruled out since there were some places with no overlap of the two channels (highlighted with the yellow arrows in Fig. 4B). Besides, hitchhiking interactions (27) between ER-mitochondria (Fig 4C, D), ER-vesicles (white arrowheads in fig. S11C), and ER themselves (magenta arrowheads in fig. S11C) were observed in our experiments (Movies S2 and S3).

\section{Labeling living tissues.} harbor a greater challenge. Tissues in vitro die faster than cultured cells without the support from the blood flow, resulting in faster mitochondrial potential gradient lost. As a consequence, the cationic dyes cannot affine to mitochondria. To permit sufficient staining deep into the living tissue, the fluorescent probes should permeate through the tissues fast enough so that they can bind to mitochondria before the gradient lost. Taking the brain slice from the mice for example, the brain slices were incubated with Atto $647 \mathrm{~N}$ immediately after the mice were sacrificed. Our results suggest that $0.5-\mathrm{h}$ incubation was sufficient enough for the dye to permeate for at least $20-\mu \mathrm{m}$ thickness, indicating its excellent permeability through tissues (Fig. 4E and Movie S4). In contrast, MitoTracker Green failed to stain the brain sample (Fig. 4F), which suggests the abilities of Atto $647 \mathrm{~N}$ to substitute MitoTracker probes in staining living tissues. Compared with the immunofluorescence techniques in previous works (28), our method requires fewer protocol steps, and the direct incubation used here avoids the structural change caused by fixation. Therefore, our method is more advantageous for many applications that expect 
166 instant actions, such as the rapid medical diagnoses of biospecimen in vitro and assessment for some 167 mitochondrial diseases $(29,30)$.

\section{Discussion}

In summary, the capability of many fluorescent dyes, which was ignored to some extent before, to stain various types of subcellular structures in living specimens with high brightness and photostability is confirmed. Labeled structures include mitochondria, ER, endocytic vesicles, and the plasma membrane. The implementation requires only specific incubation conditions without any chemical modification or physical penetration, thereby minimizing the damages and artifacts induced during the sample preparation. Moreover, Atto 647N exhibited extraordinary brightness and photostability in livecell mitochondrial labeling, which can substitute $\mathrm{SiR}$ dye in long-term imaging or super-resolution microscopy.

Due to the limits from objective conditions, especially that the structures of many of the commercially available fluorescent dyes are trade secret, not all of them are testified in this paper. However, the hypothesis built in this work gives a guideline to find these candidates out and to broaden the applications of existing dyes. Moreover, the phenomena observed here indicate their great potential to answer a wide range of biological questions in living cells and tissues.

\section{Materials and Methods}

\section{Primary cultural astrocytes}

Primary cultural astrocytes were obtained from Sprague-Dawley (SD) rat brains (1 day old). The rats were bought from Zhejiang Research Center of Laboratory Animals, China, sterilized with 75\% ethanol and sacrificed by cutting off their heads. The following steps were all done on the ice. The scalps and skulls were incised, and the brains were taken out into pre-cooling Phosphate buffered saline (PBS; Thermo Fisher Scientific, Inc.). Then the cortex was isolated, cut into pieces using knives, and incubated with pre-warmed Trypsin-EDTA (Thermo Fisher Scientific, Inc.) at $37^{\circ} \mathrm{C}$ for $20 \mathrm{~min}$. The mixture was centrifuged for $5 \mathrm{~min}$ at $1000 \mathrm{rpm}$, and the supernatant was removed. Then the cells were dissociated by adding $5 \mathrm{~mL}$ of pre-warmed growth medium (DMEM, high glucose $+10 \%$ fetal bovine serum (FBS); Thermo Fisher Scientific, Inc.) and vigorous pipetting. Finally, the cell suspension was 
incubated in a T25 flask (Thermo Fisher Scientific, Inc.) at $37^{\circ} \mathrm{C}$ in a humidified $5 \% \mathrm{CO}_{2}$ environment. The medium was changed every two days, and after 7 8 days, the culture flask was shaken manually for $30 \mathrm{~min}$ to remove the overlaying microglia exposed on the astrocyte layer. The supernatant containing microglia was discarded, and $5 \mathrm{~mL}$ of culture medium was added into the flask. This step was repeated for once to remove oligodendrocyte precursor cells.

\section{Cell culture}

HeLa (human adenocarcinoma cell line), HEK293 (human embryonic kidney epithelial cell line), NIH/3T3 (mouse embryonic fibroblast cell line), and U2OS (human osteosarcoma cell line) cells were purchased from the American Type Culture Collection. HeLa, HEK293, and NIH/3T3 cells were cultured in the DMEM medium (Thermo Fisher Scientific, Inc.). U2OS cells were cultured in McCoy's 5A medium (Thermo Fisher Scientific, Inc.). All media were supplemented with 10\% (v/v) FBS, and the cultures were maintained at $37^{\circ} \mathrm{C}$ in a humidified $5 \% \mathrm{CO}_{2}$ environment.

\section{Transfection}

Cells were grown overnight in $24-w e l l$ plates at $37^{\circ} \mathrm{C}$ in a $5 \% \mathrm{CO}_{2}$ atmosphere. After reaching over 80\% confluence, the plasmids mRuby-Clathrin (Addgene \#55852), pEGFP-Sec23A (Addgene \#66609), ZsGreen-Rab5 (custom synthesized by Genomeditech (Shanghai, China) Co., Ltd.), EGFP-Rab7A (Addgene \#28047), GFP-LAMP1 (Addgene \#16290), EMTB-3XGFP (Addgene \#26741), pSNAPfCox8A (Addgene \#101129), or pSNAPf-TOMM20 (custom synthesized by Genomeditech (Shanghai, China) Co., Ltd.) was transfected into the cells using Lipofectamine 3000 (Thermo Fisher Scientific, Inc.) according to the manufacturer's instructions. After $24 \mathrm{~h}$, the transfected cells were digested with trypsin-EDTA and seeded into Nunc Glass Bottom Dishes ( $12 \mathrm{~mm}$, Thermo Fisher Scientific, Inc.) at a density of $1.5 \sim 2.0 \times 104$ per well in growth medium $(150 \mu \mathrm{L})$. The cells were grown for an additional 12 24 $\mathrm{h}$ before incubation with the indicated probes.

\section{Live-cell labeling with commercially available probes}

Before staining, the indicated cells were seeded in Nunc Glass Bottom Dishes at a density of $1.5 \sim 2.0 \times 10^{4}$ per well in growth medium $(150 \mu \mathrm{L})$. After overnight incubation, the cells were washed three times with PBS. Work solutions of the indicated probes at different concentrations were prepared 
with phenol red-free DMEM (Thermo Fisher Scientific, Inc.). The cells were then incubated with MitoTracker Deep Red FM (200-500 nM, $100 \mu \mathrm{L}$; Thermo Fisher Scientific, Inc.), MitoTracker Green FM (1000 nM, $100 \mu \mathrm{L}$; Thermo Fisher Scientific, Inc.), SiR-actin (Cytoskeleton, Inc.) in a 5\% $\mathrm{CO}_{2}$ atmosphere at $37^{\circ} \mathrm{C}$ for $30 \mathrm{~min}$. For SNAP-Cell 647-SiR (New England Biolabs, Inc.) dye labeling, transfection of pSNAPf-Cox8A or pSNAPf-TOMM20 was performed before incubation of SNAP-Cell 647-SiR $(3 \mu \mathrm{M})$ at $37^{\circ} \mathrm{C}$ for $30 \mathrm{~min}$. After incubation, the supernatant was discarded, and a solution of Trypan blue (TB, $100 \mu \mathrm{L}, 1 \mathrm{mg} \mathrm{mL}{ }^{-1}$; Sigma-Aldrich Co., LLC) in PBS was added to exclude the dead cells and quench the extracellular fluorescence from the probes bound to either the cell membrane or the dish surface $(7,16,17)$. After $1 \mathrm{~min}$, TB was removed, and the cells were washed twice gently with PBS. Cells were immersed in Live Cell Imaging Solution (Thermo Fisher Scientific, Inc.) before imaging. ProLong Live Antifade Reagent (Thermo Fisher Scientific, Inc.) was added to this solution according to the manufacturer's instructions when required.

\section{Live-cell labeling with fluorescent dyes}

Before staining, the indicated cells were seeded in Nunc Glass Bottom Dishes at a density of $1.5 \sim 2.0 \times 10^{4}$ per well in growth medium $(150 \mu \mathrm{L})$. After overnight incubation, the cells were washed three times with PBS. Aliquots of Atto 495, Atto 565, Atto 590, Atto 647N (Sigma-Aldrich Co., LLC), BODIPY 650/665, Alexa Fluor 647 (Thermo Fisher Scientific, Inc.), Cy3B, and Cy5 (GE Healthcare Co., Ltd) NHS esters were dissolved in Dimethyl sulfoxide (DMSO; Sigma-Aldrich Co., LLC) to make 3-mM stock solutions at $-20^{\circ} \mathrm{C}$. Stock solutions were diluted with phenol red-free DMEM to work solutions at different concentrations before use. The cells were incubated with these dyes either at $37^{\circ} \mathrm{C}$ in a $5 \% \mathrm{CO}_{2}$ atmosphere or at $20^{\circ} \mathrm{C}$ for $30 \mathrm{~min}$. The post-incubation treatment was the same as that of the commercially available probes above.

\section{Labeling living tissue slices with fluorescent probes}

All animal procedures were conducted in compliance with the guidelines for animal care and use of Zhejiang University and conformed the Guide for the Care and Use of Laboratory Animal published by the National Academy Press (Washington, DC, 1996). The 12-week-old Institute of Cancer Research (ICR) mice were obtained from Zhejiang Academy of Medical Sciences [License Number: SCXK (Zhe) 2014001] and housed in cages under a standard condition of temperature $\left(23 \pm 2^{\circ} \mathrm{C}\right)$, relative humidity 
(55 $\pm 5 \%)$ and light $12 / 12 \mathrm{~h} \mathrm{light/dark} \mathrm{cycle.} \mathrm{The} \mathrm{mice} \mathrm{were} \mathrm{free} \mathrm{to} \mathrm{access} \mathrm{to} \mathrm{food} \mathrm{and} \mathrm{water.} \mathrm{Before} \mathrm{the}$ experiment, the mice were executed by euthanasia. The brains were separated and washed with cold PBS buffer. Then the brains were cut into slices from different orientations and incubated with Atto $647 \mathrm{~N}(15 \mu \mathrm{M})$ for $30 \mathrm{~min}$ at $37^{\circ} \mathrm{C}$. After incubation, the supernatant was discarded, and the tissue slices were washed twice gently with PBS. Then the tissue slices were put on the Nunc Glass Bottom Dishes with wound surface toward the glass bottom.

\section{MTS assay}

The cytotoxicity of the Atto dyes on different cell lines was tested using the MTS assay (21). Cells (3-4 $\times 10^{3}$ cells per well) were seeded into a 96-well plate and cultured in growth medium for $24 \mathrm{~h}$. The cells were incubated with the dyes $(1.5 \mu \mathrm{M}$ or $15 \mu \mathrm{M})$ in a $5 \% \mathrm{CO}_{2}$ atmosphere at $37^{\circ} \mathrm{C}$ for $30 \mathrm{~min}$, washed twice gently with PBS, and immersed in $100 \mu \mathrm{L}$ of growth medium and $20 \mu \mathrm{L}$ of CellTiter 96 AQueousOne Solution Reagent (Promega Co.). After another incubation for $3 \mathrm{~h}$ at $37^{\circ} \mathrm{C}$ in a $5 \% \mathrm{CO}_{2}$ atmosphere, the absorbance was recorded at $492 \mathrm{~nm}$ using a TECAN GENios Plus ELISA reader (Tecan, Inc.). The cell viabilities were expressed as the percentage of the A492 of the dye-treated cells (after subtracting the values of the dyes, which was tested in cells without MTS treats) to the untreated controls, and all of the measurements were performed in triplicate.

\section{Confocal laser scanning microscopy}

The confocal images were obtained using a $\mathrm{C} 2$ confocal laser scanning microscope (Nikon, Inc.) equipped with a 100×/1.49 numerical aperture oil immersion objective lens and were analyzed with NIS-elements (Nikon, Inc.) and ImageJ software (National Institutes of Health).

\section{STED microscopy}

The STED images were obtained using a STEDYCON microscope (Abberior, GmbH.) equipped with a 100×/1.49 numerical aperture oil immersion objective lens and were analyzed with ImageJ software.

\section{Statistical Analysis}


Statistical analysis was performed using Microsoft Excel 2019 (Microsoft Co., Ltd). Averages were represented as mean $\pm \mathrm{SD}$, and the number of replicates was indicated in respective figures and figure legends.

\section{References and Notes}

1. Miyawaki, A., Sawano, A. \& Kogure, T. Lighting up cells: labelling proteins with fluorophores. Nat. Cell Biol. 5, S1-S7 (2003).

2. Specht, E. A., Braselmann, E. \& Palmer, A. E. A critical and comparative review of fluorescent tools for live-cell imaging. Annu. Rev. Physiol. 79, 93-117 (2017).

3. Mishin, A. S., Belousov, V. V., Solntsev, K. M. \& Lukyanov, K. A. Novel uses of fluorescent proteins. Curr. Opin. Chem. Biol. 27, 1-9 (2015).

4. Lukinavičius, G., Reymond, L. \& Johnsson, K. Site-specific Protein Labeling: Methods and Protocols. (ed. Gautier, A. \& Hinner, M. J.) 107-118 (Humana Press, 2015).

5. Chazotte, B. Labeling Mitochondria with MitoTracker Dyes. Cold Spring Harb. Protoc. 8, 990992 (2011).

6. Fernández-Suárez, M. \& Ting, A. Y. Fluorescent probes for super-resolution imaging in living cells. Nat. Rev. Mol. Cell Biol. 9(12), 929-943 (2008).

7. Han, Y., Li, M., Qiu, F., Zhang, M. \& Zhang, Y.-H. Cell-permeable organic fluorescent probes for live-cell long-term super-resolution imaging reveal lysosome-mitochondrion interactions. Nat. Commun. 8, 1307 (2017).

8. Shen, S.-L., et al. A near-infrared lysosomal pH probe based on rhodamine derivative. Sensor. Actuat. B. Chem. 256, 261-267 (2018).

9. Jaiswal, J. K., Mattoussi, H., Mauro, J. M., \& Simon, S. M. Long-term multiple color imaging of live cells using quantum dot bioconjugates. Nat. Biotechnol. 21, 47-51 (2003).

10. Ji, N., Freeman, J., \& Smith, S. L. Technologies for imaging neural activity in large volumes. Nat. Neurosci. 19, 1154-1164 (2016).

11. Donaldson, J. G. Immunofluorescence staining. Curr. Protoc. Cell Biol. 69, 4-3 (2015). 
12. Hennig, S., et al. Instant Live-Cell Super-Resolution Imaging of Cellular Structures by Nanoinjection of Fluorescent Probes. Nano Lett. 15, 1374-1381 (2015).

13. Erazo-Oliveras, A., et al. Protein delivery into live cells by incubation with an endosomolytic agent. Nat. Methods 11, 861-867 (2014).

14. $\mathrm{Xu}, \mathrm{W} ., \mathrm{Z}$ Zeng, Z., Jiang, J.-H., Chang, Y.-T. \& Yuan, L. Discerning the chemistry in individual organelles with small $\square$ molecule fluorescent probes. Angew. Chem.-Int. Edit. 55, 13658-13699 (2016).

15. Cottet $\square$ Rousselle, C., Ronot, X., Leverve, X. \& Mayol, J.-F. Cytometric assessment of mitochondria using fluorescent probes. Cytom. Part A 79, 405-425 (2011).

16. Manceur, A., Wu, A. \& Audet, J. Flow cytometric screening of cell-penetrating peptides for their uptake into embryonic and adult stem cells. Anal. Chem. 364, 51-59 (2007).

17. Sahlin, S., Hed, J. \& Runfquist, I. Differentiation between attached and ingested immune complexes by a fluorescence quenching cytofluorometric assay. J. Immunol. Methods 60(1-2), 115-124 (1983).

18. Wories, H. J., Koek, J. H., Lodder, G. \& Lugtenburg, J. A novel water $\square$ soluble fluorescent probe: Synthesis, luminescence and biological properties of the sodium salt of the 4-sulfonato-3, 3', 5, 5'tetramethyl-2, 2'-pyrromethen-1, 1'-BF2 complex. Recl. Trav. Chim. Pays-Bas 104, 288-291 (1985).

19. Simons, M., et al. Electrochemical cues regulate assembly of the Frizzled/Dishevelled complex at the plasma membrane during planar epithelial polarization. Nat. Cell Biol. 11, 286-294 (2009).

20. Friedman, J. R. \& Nunnari, J. Mitochondrial form and function. Nature 505, 335-343 (2014).

21. Cory, A. H., Owen, T. C., Barltrop, J. A. \& Cory, J. G. Use of an aqueous soluble tetrazolium/formazan assay for cell growth assays in culture. Cancer Commun. 3, 207-212 (1991).

22. Riss, T. L. \& Moravec, R. A. Comparison of MTT, Xtt, and a novel tetrazolium compound for MTS for in vitro proliferation and chemosensitivity assays. Mol. Biol. Cell 3, 184a (1992).

23. Sun, J. Molecular Regulation of Endocytosis. (ed. Ceresa, B.) 61-64 (InTechOpen, 2012). 
24. Westbrook, A. W., Ren, X., Moo-Young, M., \& Chou, C. P. Engineering of cell membrane to enhance heterologous production of hyaluronic acid in Bacillus subtilis. Biotechnol. Bioeng. 115, 216-231 (2018).

25. Huang, X., et al. Fast, long-term, super-resolution imaging with Hessian structured illumination microscopy. Nat. Biotechnol. 36, 451-459 (2018).

26. Stephan, T., Roesch, A., Riedel, D. \& Jakobs, S. Live-cell STED nanoscopy of mitochondrial cristae. Sci. Rep. 9, 12419 (2019).

27. Guo, Y., et al. Visualizing intracellular organelle and cytoskeletal interactions at nanoscale resolution on millisecond timescales. Cell 175, 1430-1442 (2018).

28. Gasser, E. M. S., et al. Immunofluorescence in brain sections: simultaneous detection of presynaptic and postsynaptic proteins in identified neurons. Nat. Protoc. 1, 1887-1897 (2006).

29. Lee, J. H., et al. The Role of Adipose Tissue Mitochondria: Regulation of Mitochondrial Function for the Treatment of Metabolic Diseases. In. J. Mol. Sci. 20, 4924 (2019).

30. Chakrabarty, S., et al. Mitochondria in health and disease. Mitochondrion 43, 25-29 (2019).

\section{Acknowledgments}

We thank Mr. Yisheng Wu from SRstar Instruments Ltd., Shanghai, China, for operating the Abberior STEDYCON. This work was financially sponsored by the grants from National Key R\&D Program of China (2018YFA0701400), the National Natural Science Foundation of China (61827825, 61735017, and 31901059), Fundamental Research Funds for the Central Universities (2019XZZX00306 and 2019QNA5006), China Postdoctoral Science Foundation (2019M662042), Natural Science Foundation of Zhejiang province (LR16F050001), Zhejiang Lab (2018EB0ZX01), and ZJU-Sunny Photonics Innovation Center (2019-01).

Author contributions: X. Hao, Y. Han, C. Kuang, and X. Liu conceived of the project. Experiments were performed primarily by Y. Han. Z. Zhang, W. Liu, Y. Chen, and W. Wang set up the system and contributed to the imaging. Y. Han, Y. Yao, and X. Luo prepared the samples. Y. Han and X. Hao drafted the manuscript. All authors contributed to the manuscript polish. 
352 Competing interests: The authors declare that there are no conflicts of interest related to this 353 article.

354 Data and materials availability: The authors declare that all data supporting the findings of this 355 study are available within the article and its Supplementary Information files or from the corresponding 356 author X. H. (haox@zju.edu.cn) on reasonable request. 

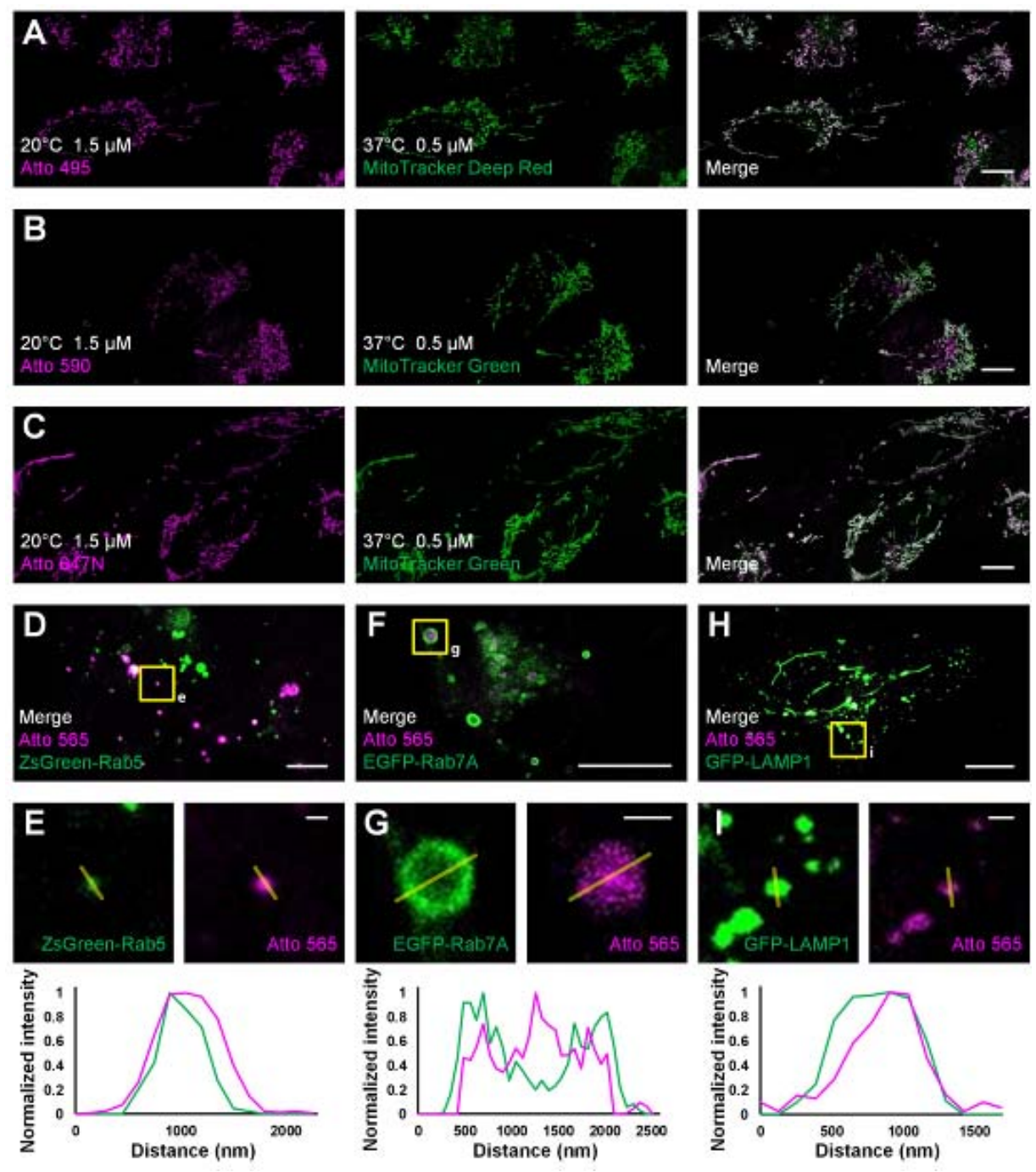

Fig. 1. Co-localization studies employing different fluorescent probes and proteins as the standard mitochondrial markers. (A) Living U2OS cells were incubated with Atto 495 (magenta, $1.5 \mu \mathrm{M}$ ) for

$36130 \mathrm{~min}$ at $20^{\circ} \mathrm{C}$ and then with MitoTracker Deep Red (green, $0.5 \mu \mathrm{M}$ ) for $30 \mathrm{~min}$ at $37^{\circ} \mathrm{C}$ before

362 imaging. Living U2OS cells were incubated with (B) Atto 590 or (C) Atto 647N (magenta, $1.5 \mu \mathrm{M}$ ) for $36330 \mathrm{~min}$ at $20^{\circ} \mathrm{C}$ and then with MitoTracker Green (green, $0.5 \mu \mathrm{M}$ ) for $30 \mathrm{~min}$ at $37^{\circ} \mathrm{C}$ before imaging. 364 Living U2OS cells transiently transfected by (D) ZsGreen-Rab5 (green; early endosomes), (F) EGFP365 Rab7A (green; late endosomes), and (H) GFP-LAMP1 (green; lysosomes) were stained with Atto 565 366 (magenta, $6 \mu \mathrm{M}$ ) for $30 \mathrm{~min}$ at $37^{\circ} \mathrm{C}$ and imaged by confocal microscope. (E, G, I) Enlarged images 367 from the boxed regions shown in $\mathrm{d}, \mathrm{f}, \mathrm{h}$, respectively. Profiles represent the fluorescence intensities 368 across the yellow lines in green and magenta channels. Scale bars, $(\mathbf{A}-\mathbf{C}, \mathbf{D}, \mathbf{F}, \mathbf{H}) 10 \mu \mathrm{m}$, and $(\mathbf{E}, \mathbf{G}, \mathbf{I})$ $3691 \mu \mathrm{m}$. 

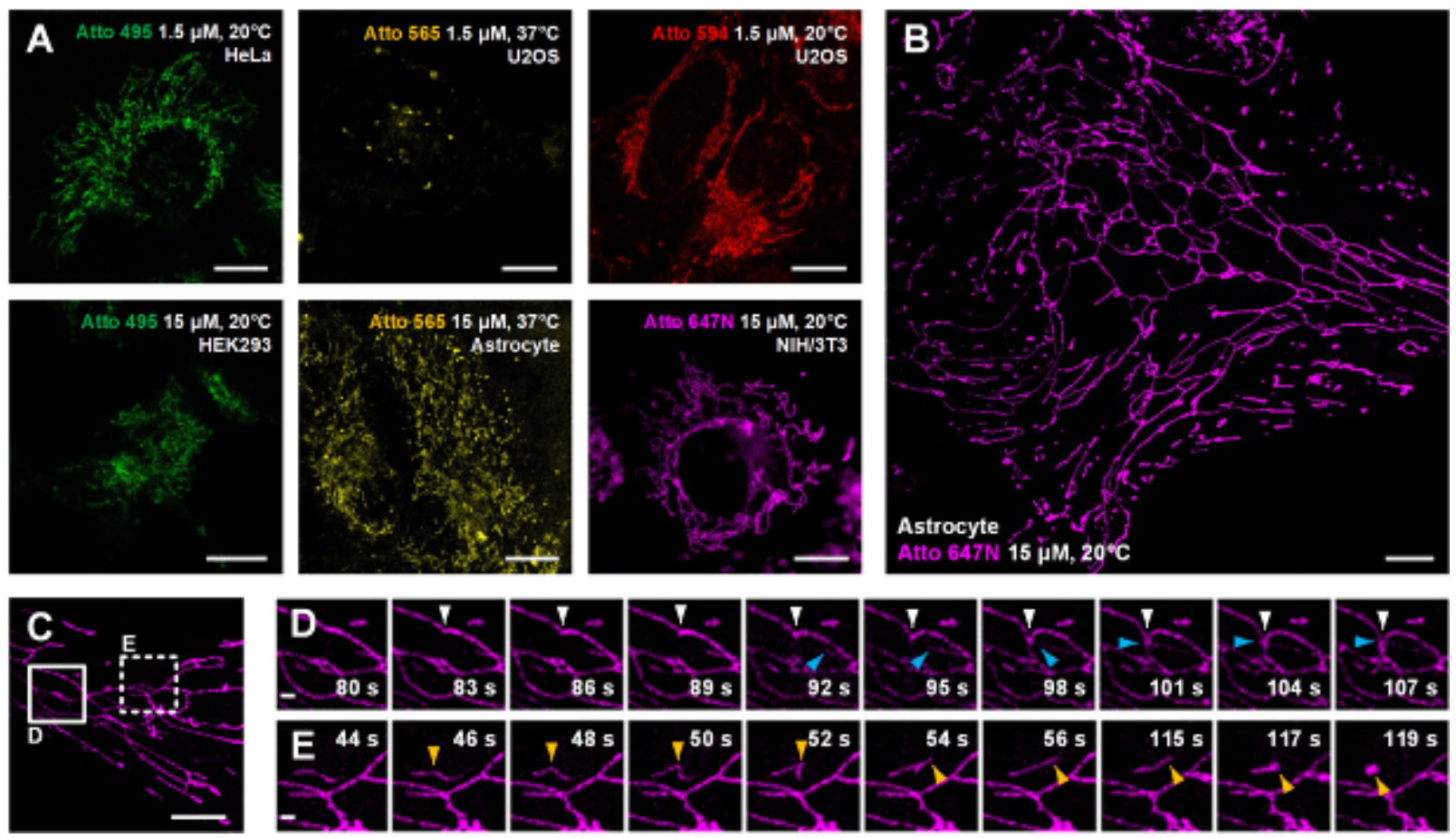

Fig. 2. Various types of living cells stained with the Atto dyes. (A) Confocal images of living U2OS,

372 Astrocyte, H3K293, HeLa, and NIH/3T3 cells incubated with the indicated Atto dyes at either $37^{\circ} \mathrm{C}$ or

$37320^{\circ} \mathrm{C}$. (B, C) Living Astrocyte cells stained with Atto $647 \mathrm{~N}$ at $20^{\circ} \mathrm{C}$ and imaged with a confocal

374 microscope. (D, E) Enlarged time-lapse images from the solid and dashed boxed regions shown in c. No

375 deconvolution or bleaching compensation procedure was applied during the figure rendering. For the

376 time-lapse images, consecutive frames spaced at 1-s intervals were obtained; representative images of

377 consecutive frames are displayed (more frames are shown in Movie S1). Scale bars, (A-C) $10 \mu \mathrm{m}$, and

$378(\mathbf{D}, \mathbf{E}) 1 \mu \mathrm{m}$. 

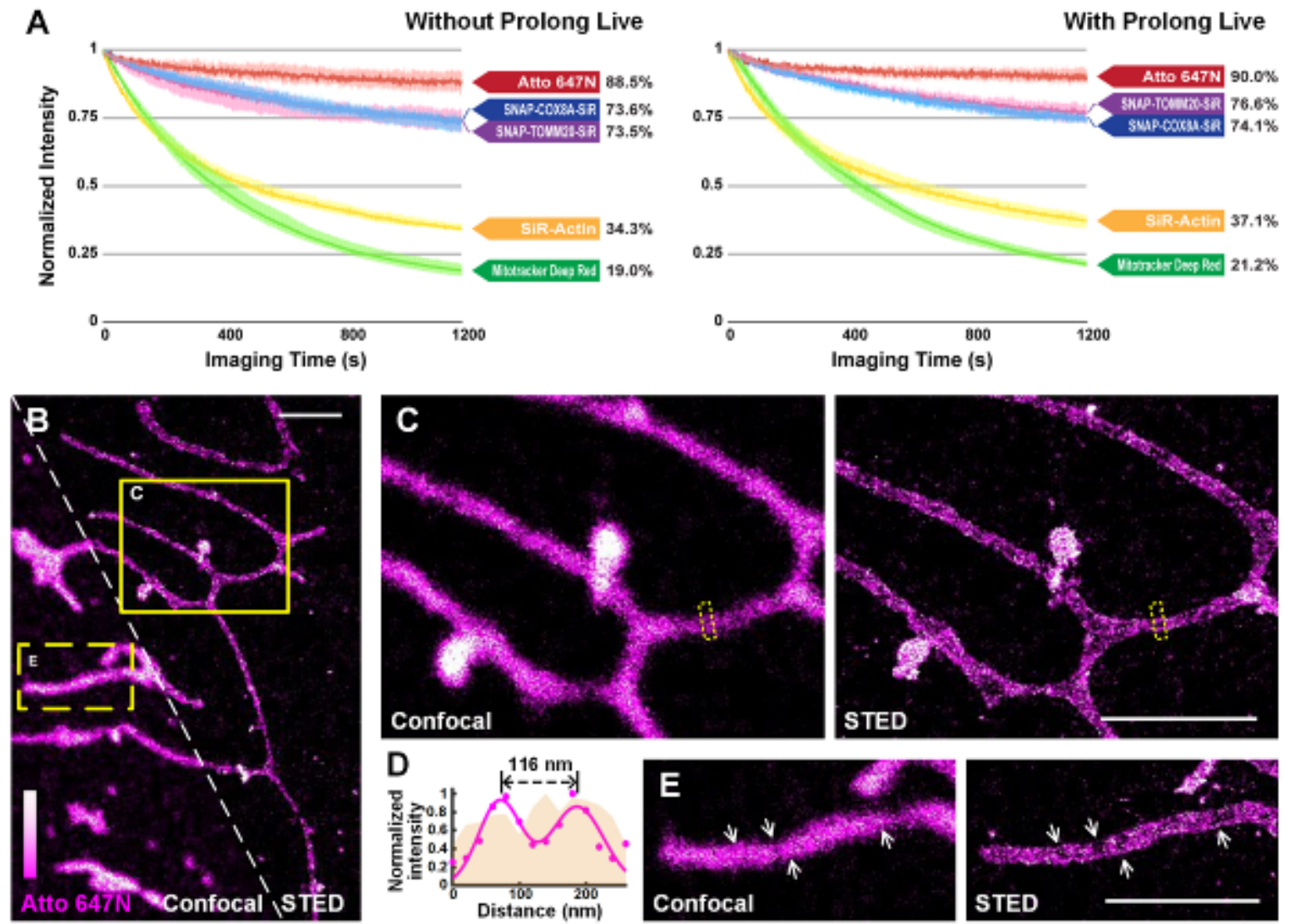

Fig. 3. Atto 647N for live-cell imaging applications with high demand for optical properties. (A)

Curves of intensity decay of the indicated dyes without (left) or with (right) the addition of Prolong Live under the same confocal imaging condition. Consecutive frames spaced at 1-s intervals were recorded. or $(\mathbf{E})$ dashed yellow boxes shown in $b$.

(D) Intensity profiles at the positions within the dashed boxes in

Scale bars, $2 \mu \mathrm{m}$. 

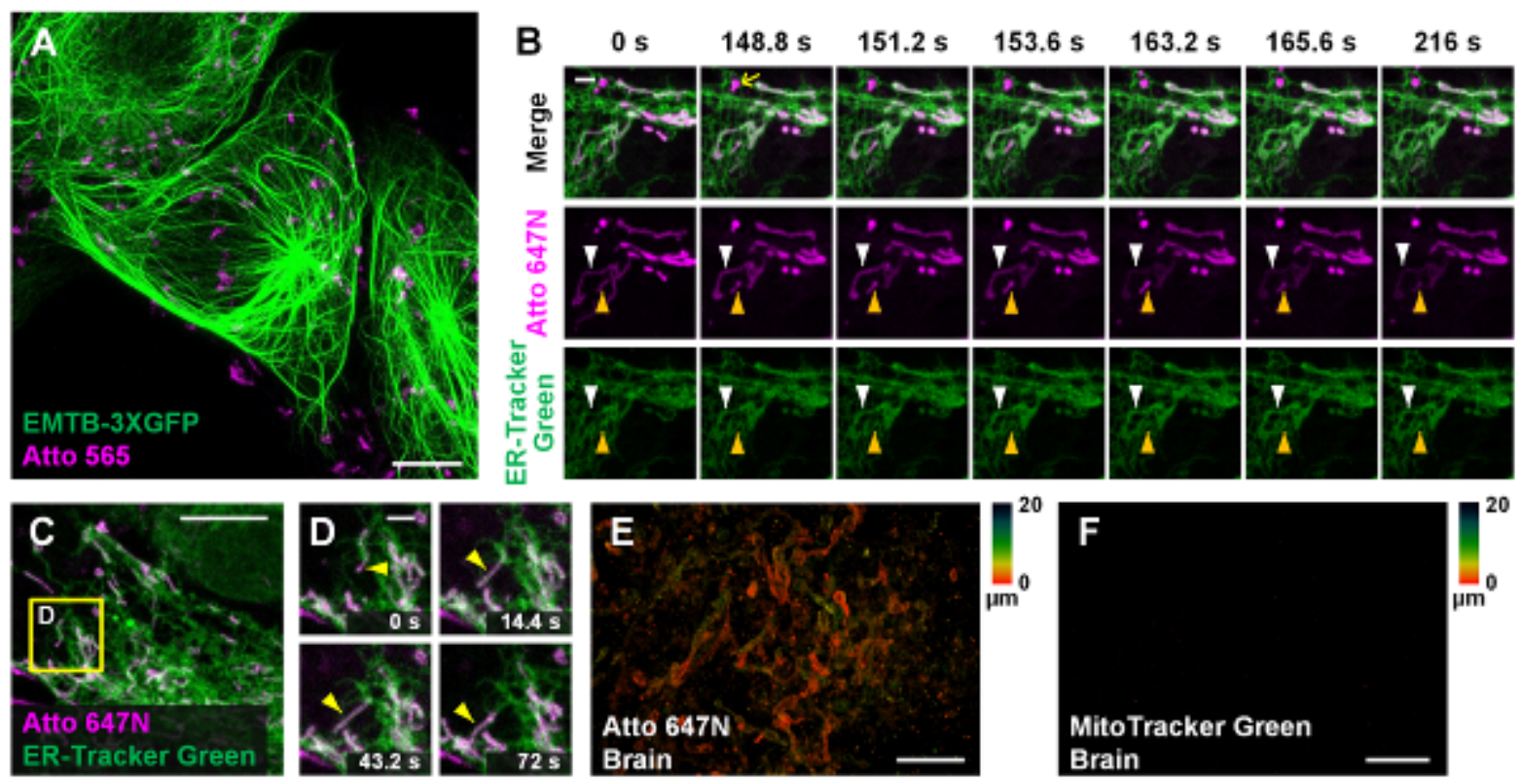

Fig. 4. Other applications in living cells and tissues. (A) Dual-color confocal image (maximum intensity projection) of living U2OS cells labeled with EMTB-3XGFP (green) and Atto 565 (magenta; 3 $\mu \mathrm{M}$ ). (B, C) Dual-color confocal image of living Astrocytes labeled with Atto 647N (magenta; $15 \mu \mathrm{M}$, middle row) and ER-Tracker Green (green; $2 \mu \mathrm{M}$, bottom row) at for $30 \mathrm{~min}$ at $20^{\circ} \mathrm{C}$. (D) Mitochondria move along ER tubules. No deconvolution or bleaching compensation procedure was applied during the figure rendering. For the time-lapse images, consecutive frames spaced at 2.4-s intervals were recorded; representative images of consecutive frames are displayed (more frames are shown in Movies S2 and S3). Brain slices incubated with (E) Atto 647N and (F) MitoTracker Green. Color bars on the right of (E) and (F) indicate the imaging depth. Scale bars, (A, C) $10 \mu \mathrm{m},(\mathbf{B}, \mathbf{D}) 2 \mu \mathrm{m}$, and (E, F) $20 \mu \mathrm{m}$. 
399 Table 1. Recommended conditions for specifically labeling subcellular structures in living cells using 400 the live-cell incompatible dyes.

\begin{tabular}{|c|c|c|c|c|c|}
\hline $\begin{array}{c}\text { Subcellular } \\
\text { structures }\end{array}$ & Electric charges & $\begin{array}{l}\text { Additional } \\
\text { properties }\end{array}$ & $\begin{array}{c}\text { Incubation } \\
\text { concentration }\end{array}$ & $\begin{array}{c}\text { Incubation } \\
\text { temperature }\end{array}$ & $\begin{array}{c}\text { Recommended } \\
\text { dyes }\end{array}$ \\
\hline Mitochondria & $\begin{array}{c}\text { Cationic dyes, or } \\
\text { zwitterionic } \\
\text { BODIPY dyes }\end{array}$ & $\begin{array}{l}\text { Moderate cell } \\
\text { permeability }\end{array}$ & $1.5-15 \mu \mathrm{M}$ & $20^{\circ} \mathrm{C}$ & Atto $647 \mathrm{~N}$ \\
\hline$E R$ & $\begin{array}{l}\text { Cationic dyes, or } \\
\text { zwitterionic } \\
\text { BODIPY dyes }\end{array}$ & $\begin{array}{c}\text { Higher cell } \\
\text { permeabilities or } \\
\text { dosages }\end{array}$ & $15 \mu \mathrm{M}$ & $20^{\circ} \mathrm{C}$ & BODIPY 650/665 \\
\hline $\begin{array}{c}\text { Endocytic } \\
\text { vesicles }\end{array}$ & - & $\begin{array}{l}\text { Particularly low } \\
\text { cell-permeability }\end{array}$ & $1.5-15 \mu \mathrm{M}$ & $37^{\circ} \mathrm{C}$ & AF 647, Су3B \\
\hline $\begin{array}{c}\text { Plasma } \\
\text { membrane }\end{array}$ & Cationic dyes & $\begin{array}{c}\text { Especially high } \\
\text { lipophilicity }\end{array}$ & $1.5-15 \mu \mathrm{M}$ & $20^{\circ} \mathrm{C}$ & Cy5 \\
\hline
\end{tabular}




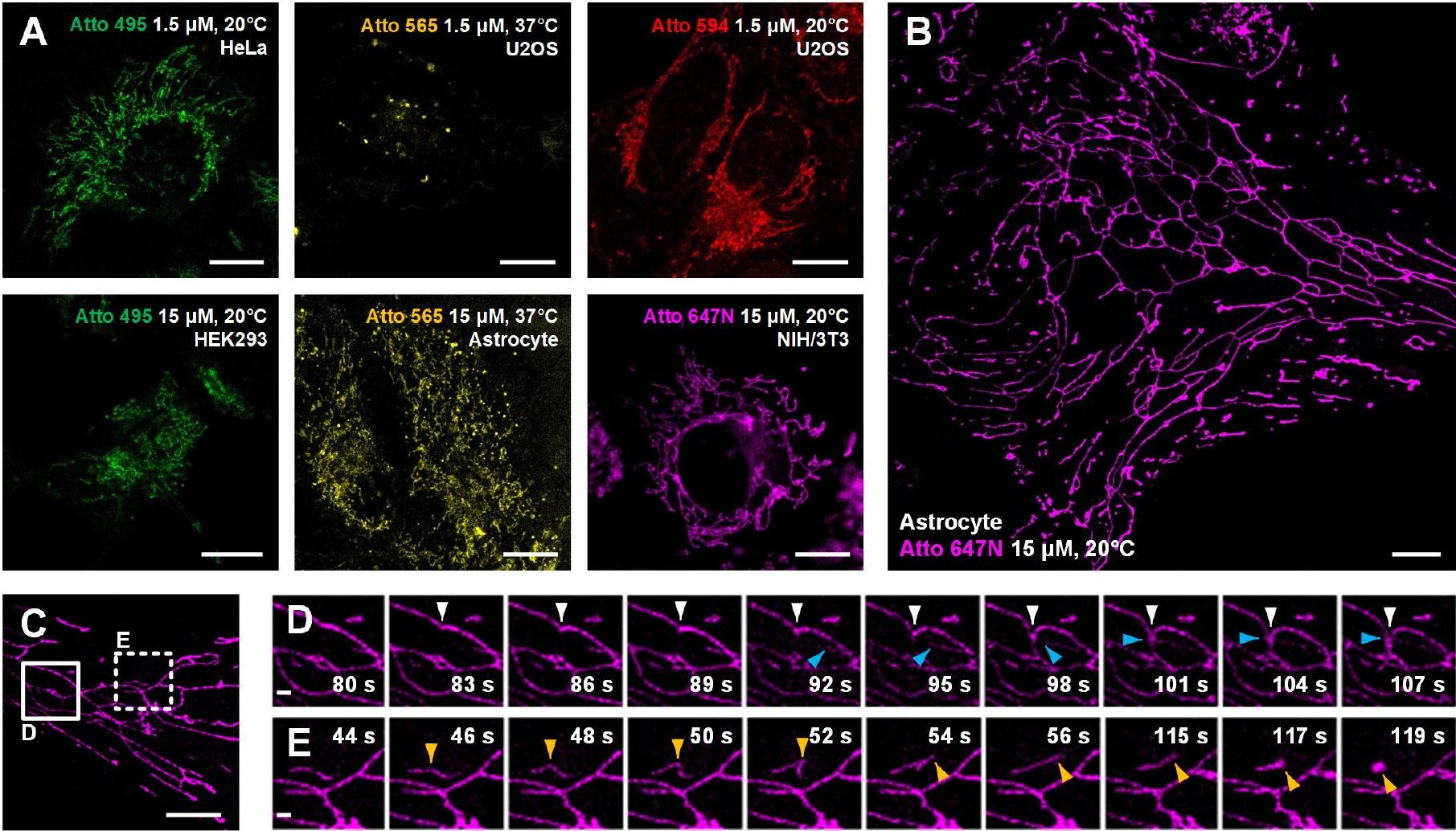




\section{A}

$20^{\circ} \mathrm{C} 1.5 \mu \mathrm{M}$

Atto 495

$37^{\circ} \mathrm{C} .0 .5 \mu \mathrm{M}$

MitoT racker Deep Red

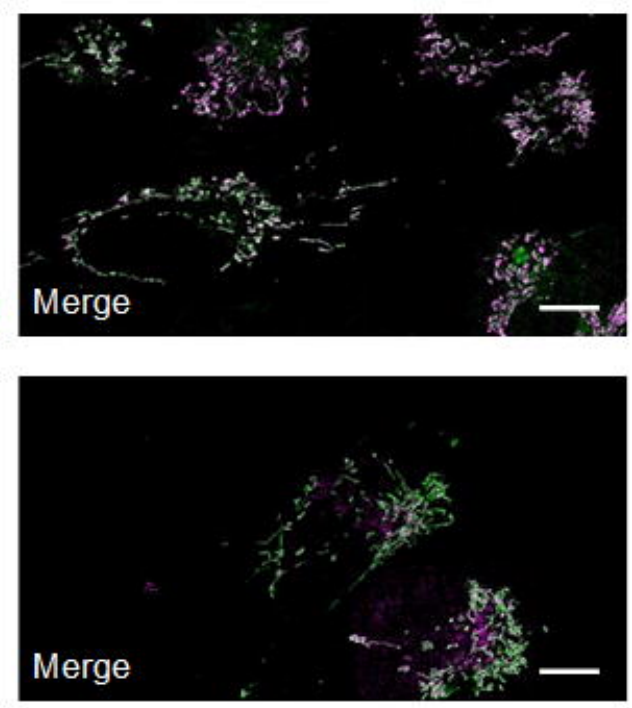

\section{C}

$20^{\circ} \mathrm{C} \cdot 1.5 \mu \mathrm{MM}$

Atto $647 \mathrm{~N}$

$37^{\circ} \mathrm{C} \cdot 0.5 \mu \mathrm{M}$

Mitof ấa ker Greên

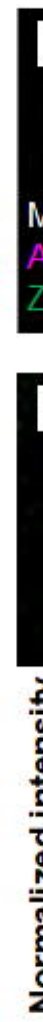

D

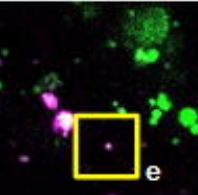

Merge

Atto 565

ZsGreen-Rab5.

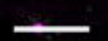

$\mathbf{F}$

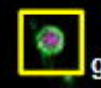

Merge

Atto 565

EGFP-Rab 7A

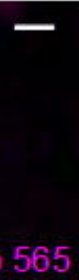

ZsGreen-Rab5

.

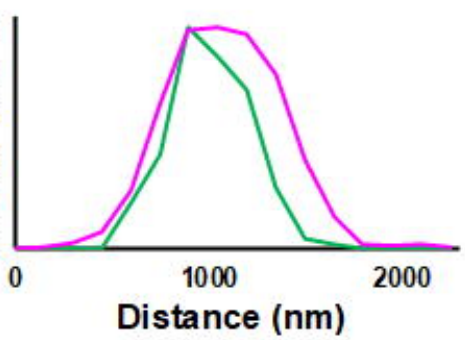

Atto 565

EGFP-Rab7A

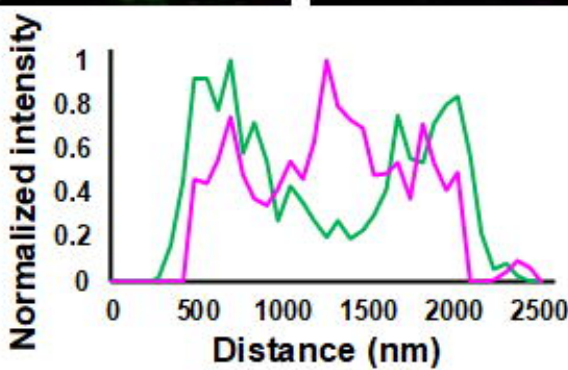

?
Merge

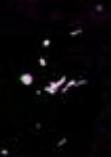

H

$\begin{array}{llll}\text { Merge } & \\ \text { Atto } 565 & \cdots & \end{array}$

GFP-LAMP1
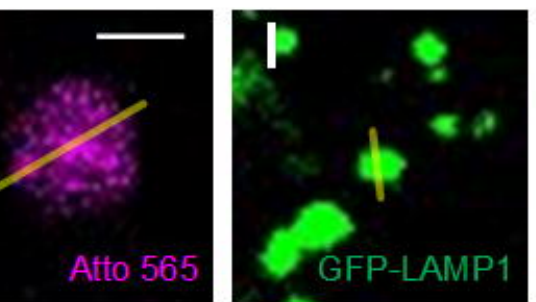

Atto 565

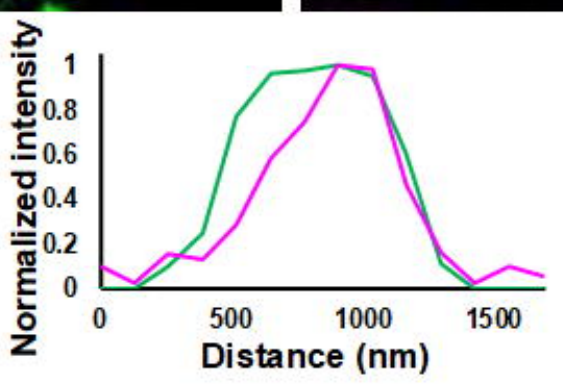




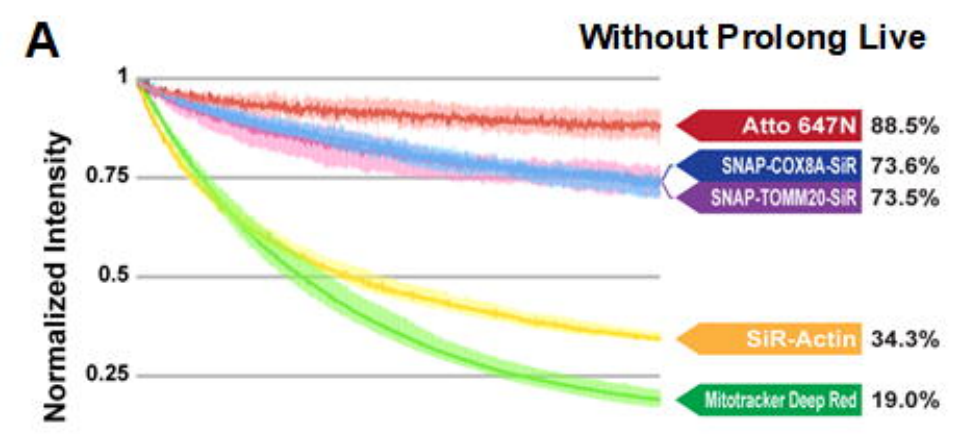

With Prolong Live
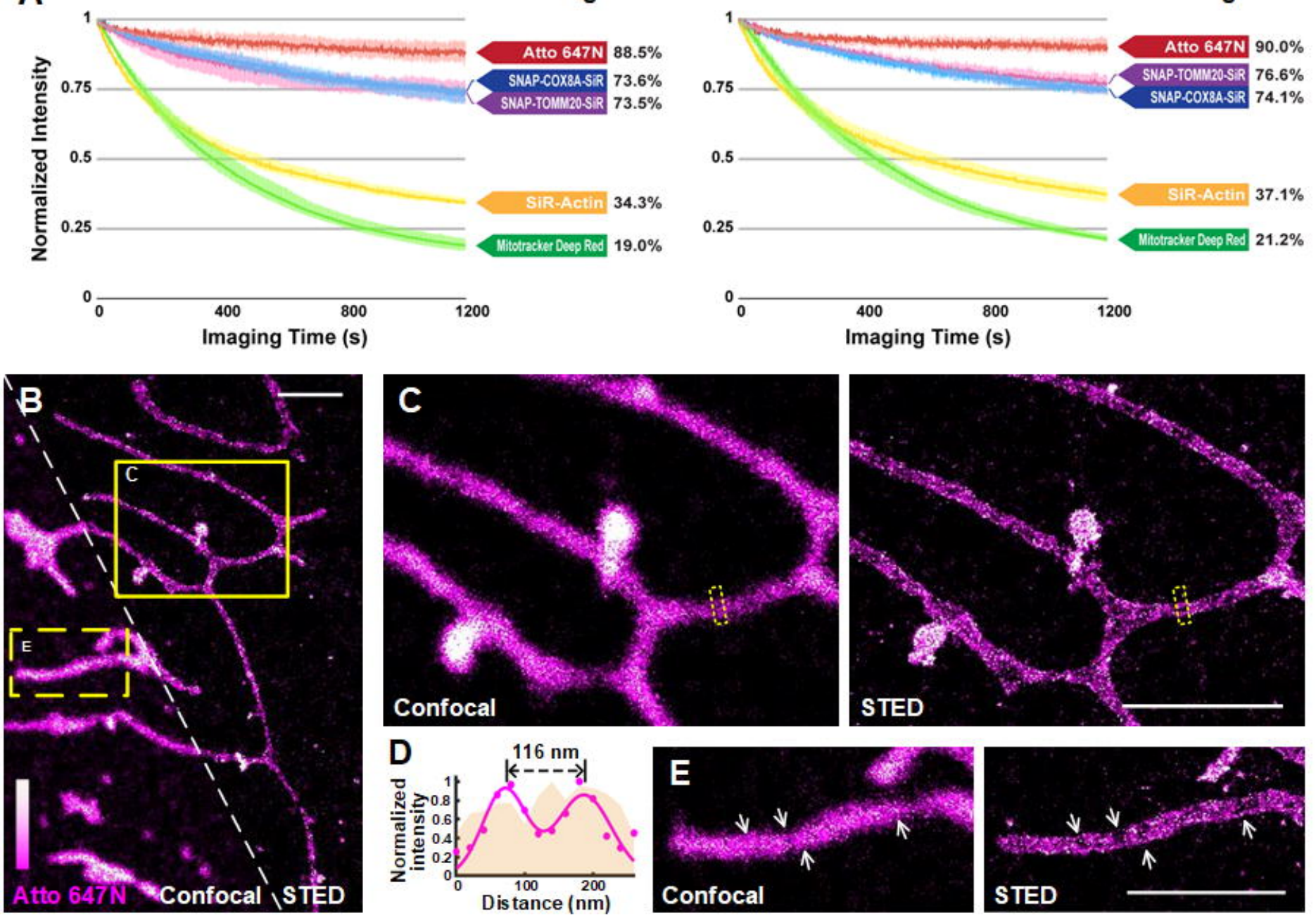